\title{
La eficacia de la vaguedad y la imprecisión léxica: cómo se obtiene el significado de la proforma eso
}

THE EFFECTIVENESS OF VAGUENESS AND LEXICAL IMPRECISION: HOW THE MEANING OF THE PROFORME ESO IS OBTAINED

\author{
Juan Carlos Tordera Yllescas
}

Universitat de València

Resumen

El pronombre eso ha sido considerado tradicionalmente como un pronombre impreciso y vago. Sin embargo, siendo un elemento tan impreciso y vago, la pregunta que nos formulamos es cómo es posible que sea tan utilizado en las conversaciones cotidianas. Si fuera así, tendríamos que concluir que nuestras conversaciones tienden a la imprecisión y a la vaguedad y no parece que este sea el objetivo del hablante, quien busca que su conversación sea lo más eficaz que sea posible. En este artículo, se muestra, por un lado, que existen mecanismos gramaticales y semántico-discursivos (v.gr.: la organización impuesta por las relaciones retóricas) que permiten desentrañar el sentido de eso. Por otro lado, se tratará de mostrar que el pronombre eso, en determinadas estructuras (v.gr.: y eso) tiene un valor ilocutivo que lo hace altamente eficaz desde el punto de vista discursivo.

Palabras clave: eso, imprecisión léxica, relaciones retóricas, pronombres

\begin{abstract}
The pronoun eso has traditionally been considered as an imprecise and vague pronoun. However, being an element so imprecise and vague, the question that should be formulated is how is possible that this pronoun is so used in the quotidian conversations. If so, it would have to be concluded that the conversations try to be imprecise and vague and, however, the goal of the speaker (who seeks his conversation to be as effective as possible) does not seem to be in that way. In this article, it is demonstrated, on the one hand, there are grammatical and semantical-discursive mechanisms (e.g.: the organization imposed by the rhetorical relations) that allow to decipher the meaning of eso. On the other hand, it is intended to show that the pronoun eso, in certain structures (e.g.: y eso) has a value illocutive that it makes highly effective from the point of view discursive.
\end{abstract}

KEYWORDS: eso, lexical imprecision, rhetorical relations, pronoun 
INTRODUCCIÓN

Si a un hablante común del español se le preguntara por el significado de la palabra eso posiblemente encontraría más problemas a la hora de definirla que otras como mesa, perro, libro... y un largo etcétera. Posiblemente, el hablante calificaría el significado de eso como vago, impreciso, inespecifico y calificativos semejantes. Sin embargo, ha de llamar la atención del lingüista el hecho de que una expresión tan vaga, tan imprecisa o tan inespecífica esté bien presente en el discurso oral cotidiano. Si dicha palabra fuera tan ambigua o tan "así", ¿por qué se utiliza tanto? Para la lógica de algunos lingüistas, expresiones de este tipo solo crearía discursos necesariamente ineficaces o discursos irrelevantes (en el sentido de Sperber y Wilson, 1986 [1994]).

Así pues, los objetivos del presente trabajo se pueden enumerar en tres sentidos:

a) Demostrar que el contexto gramatical determina en buena medida el referente de eso.

b) Explicar cómo las relaciones semántico-pragmáticas del discurso puede determinar el referente de eso.

c) Y justificar que las proformas como eso tienen una función pragmático-discursiva altamente relevante. Dicho valor se puede ejemplificar de manera clara con la expresión y eso, denominada normalmente coletilla.

Los objetivos trazados tratarán de mostrar que el significado de la "proforma"1 eso no es, en absoluto, impreciso, sino que es usado por los hablantes de manera intencional, eficaz y precisa.

\section{GRAMÁTICA Y REFERENTE PRONOMINAL}

Toda forma pronominal suele tomar su referente a partir de una expresión anterior (anáfora) o posterior (catáfora), a la que se la denomina antecedente o consecuente. El referente puede ser un individuo (v.gr.: «Juan me ha dicho que él no irá a la fiesta»), un evento (v.gr.: "Juan levantó cien kilos de peso, pero su padre no lo vio»), una propiedad (v.gr.: «Pedro es muy celoso, pero su novio no es aśr(»)... Pues bien, eso, que es la "proforma por excelencia", no solo puede referirse a individuos ( $\mathrm{x}$ ), sino también a eventos (e) $\mathrm{O}$ propiedades (f), entre otros posibles referentes ${ }^{2}$.

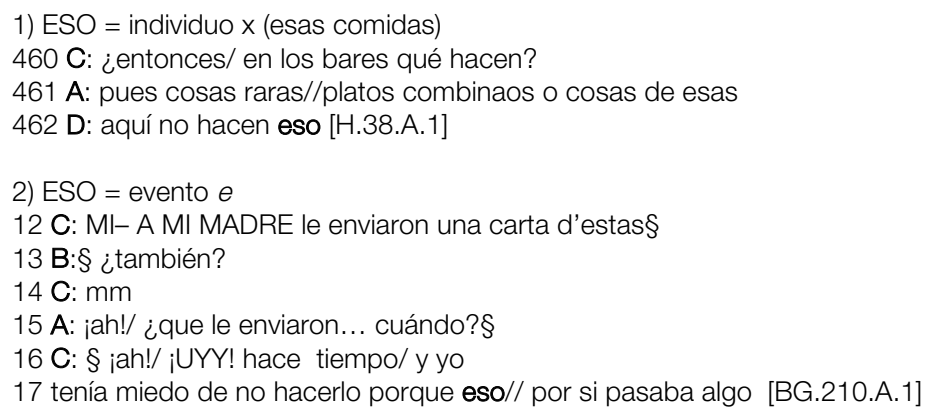

\footnotetext{
${ }^{1}$ Se entiende por proforma aquel elemento gramatical que está en sustitución de cualquier otro elemento. Como se tratará de demostrar, el uso de eso no es libre sino que obedece a unas restricciones gramaticales, semánticas y discursivas que lo incapacita para sustituir "cualquier cosa". No obstante, a falta de un mejor término consensuado en nuestra tradición (¿instructor procedimental? ¿esqueleto directivo?...), utilizaremos en el presente trabajo esta etiqueta.

2 Todos los ejemplos de nuestro corpus han sido extraído del Corpus Val.Es.Co 2.0, de consulta disponible en http://www.valesco.es/?q=es/corpus.

Normas (ISSN: 2174-7245) | 


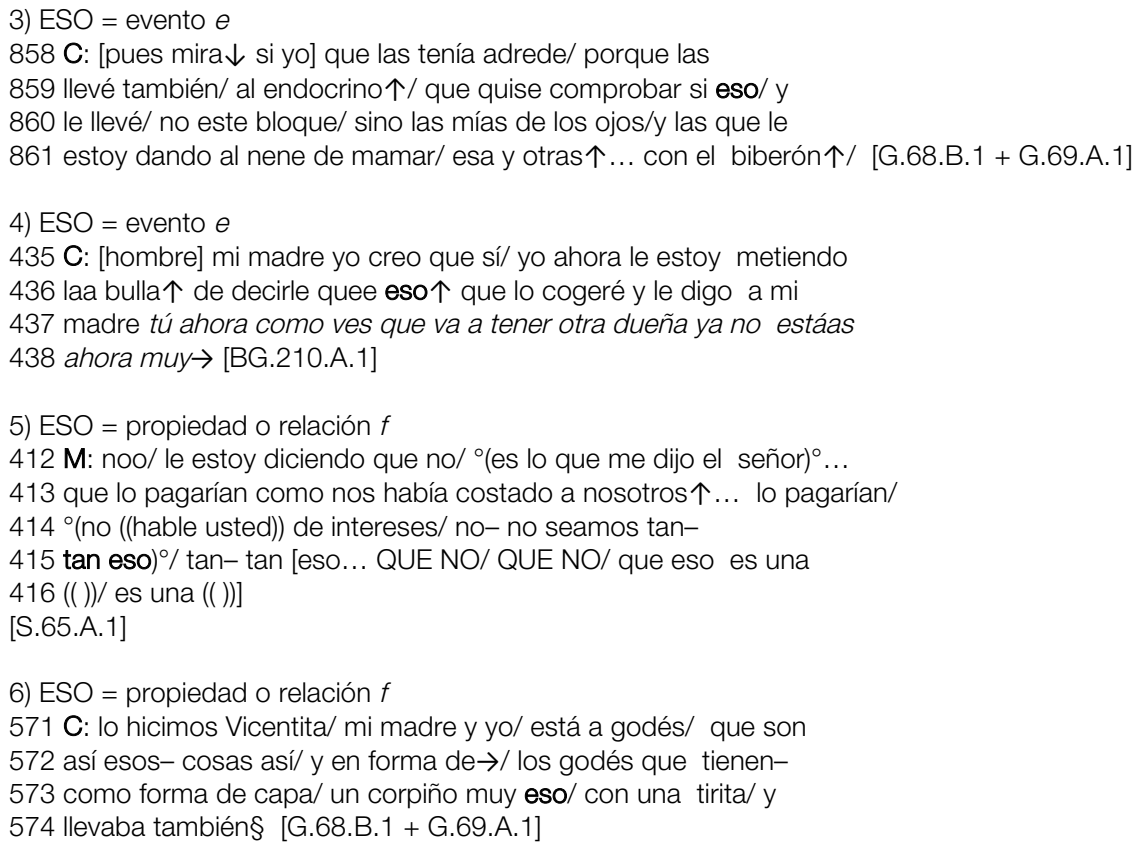

A nuestro juicio, el componente gramatical puede ofrecernos una guía parcial para desentrañar el significado de la palabra eso. Cuando dicha palabra ocupa un contexto sintáctico no prototípico (el de un SN), el referente de la proforma eso estará determinado por el contexto categorial. En concreto, se pueden establecer las siguientes reglas de asignación referencial:

\footnotetext{
Referente eventual (e): cuando ocupa una posición oracional/proposicional.

Si [eso] orac: contexto oracional; referente eventual.

Referente de propiedad/relación (f): cuando ocupa una posición adjetival

Tan [eso]adj: contexto adjetival; referente de propiedad.
}

La primera regla explicaría por qué necesariamente en los ejemplos de (2), (3) y (4), el pronombre eso tiene un referente eventual, mientras que la segunda regla da cuenta de la razón por la que, en los ejemplos de (5) y (6) dicho pronombre tiene un referente de propiedad y no puede presentar otro tipo de referente.

Un caso particular es el contexto en el que el pronombre eso aparece seguido de un artículo y ocupando el lugar del núcleo del SN. En este caso, el referente del artículo+eso solo puede ser individual y nunca eventual o de propiedad, tal como aparece en el siguiente ejemplo:

7) 693 C: $\S$ que siempre siendo la- la eso de todos/ la

694 ayudanta y tal؟ / ME ALEGRO /ME ALEGRO /Y ASI MÁS DE CUATRO [G.68.B.1 + G.69.A.1]

Sin embargo, en no pocos casos, el contexto oracional es poco relevante para determinar el referente de la proforma eso. Cuando dicho pronombre ocupa el contexto sintáctico de un $\mathrm{SN}$, el referente de este no necesariamente tiene por qué ser el referente propio de un $\mathrm{SN}$, tal como se observan de los siguientes ejemplos.

8) 9 B: jyee pasa las papas!/ ¡hostia个! medio paquete os habéis hecho ya $\downarrow$ cabrones/ déjame coger§

11 D: $\S$ medio paqu- noo de eso no se

12 llena/// (8") [dame cocacola] [H.38.A.1]

9) 1331 es que ya te digo o s(e)a fue y aprobó/// sin haber ido a la 
1332 autoescuela $\downarrow$ habiendo leído el libro [una vez=]

1333 L: [y aprobó el teórico]

$1334 \mathrm{G}:=$ yy ceporro [perdío que es=]

$1335 \mathrm{E}:[$ sii/ yaa]

1336 G: = porque mi vecino para eso [es un ceporro perdíoo] [L.15.A.2]

Mientras que, en el ejemplo de (8), el referente de la proforma eso es claramente de individuo, en cambio, en (9), más bien parece que el referente es eventual. Pero en este tipo de casos, que son numerosos, el contexto sintáctico no aporta ninguna información determinante para obtener el significado de eso. Necesitamos de una teoría semánticopragmática que nos ayude a determinar el referente de eso en sus contextos sintácticos prototípicos (cuando ocupa el lugar de un SN).

\section{EL COMPONENTE SEMÁNTICO-PRAGMÁTICO}

Desde los trabajos clásicos de Mann y Thompson (1987 y 1988), se ha aceptado que los diferentes enunciados mantienen entre sí diferentes relaciones retóricas/discursivas (v.gr.: secuencia, contraste, motivo...). Y diversos trabajos como los relacionados con la teoría Penn Discourse TreeBank (Webber, Stone, Joshi y Knott, 1994; Prasad, Dinesh, Lee, Joshi y V. Webber, 2007; Prasad, Dinesh, Lee, Miltsakaki, Robaldo, Joshi y Webber, 2008; Prasad, Joshi y Webber, 2010; entre otros muchos) o la Segmented Discourse Representation Theory (Asher, 1998 o Asher y Lascarides, 2003) han explotado la idea de las relaciones retóricas/discursivas con el fin de desentrañar el referente de los elementos anafóricos. Para estos autores, la determinación del referente pronominal está íntimamente unida a las relaciones retóricas.

En estos trabajos, se asume que existen relaciones retóricas/discursivas coordinadas y relaciones retóricas/discursivas subordinadas. El tipo de relación (subordinada o coordinada) es determinante a la hora de asignar el referente adecuado para el pronombre. Grosso modo, los elementos anafóricos que se hallan en enunciados que mantienen una relación retórica coordinada con el enunciado contiguo pueden establecer una relación fórica con este; los elementos anafóricos que se hallan en enunciados subordinados desde el punto de vista retórico/discursivo pueden acceder a cualquier referente que se halle en un enunciado jerárquicamente superior. Con el fin de ilustrar esta afirmación, se partirá del siguiente ejemplo:

10) Juan viajó primero a París $\left(p_{1}\right)$. Allí conoció a mucha gente interesante como Andrés $\left(p_{2}\right)$ y visitó diversos parajes muy bellos con él $\left(\mathrm{p}_{3}\right)$. $\mathrm{Y}$, después, $\varnothing$ viajó a Turquía $\left(\mathrm{p}_{4}\right)$.

La representación de la estructura retórica de este ejemplo respondería, aproximadamente, a la siguiente:

\section{narración}

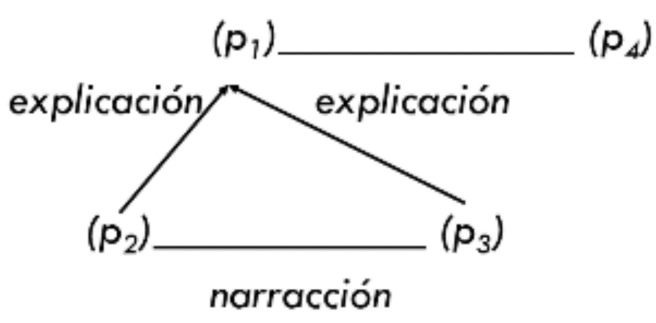

Figura 1 
El enunciado $\left(p_{3}\right)$ está subordinado a $\left(p_{1}\right)$ y está coordinado con $\left(p_{2}\right)$. Por tanto, el pronombre que aparezca en $\left(p_{3}\right)$ tendrá acceso tanto al referente que aparezca en $\left(p_{1}\right)$ (esto es, Juan) como a los referentes que aparezcan en $\left(\mathrm{p}_{2}\right)$ (esto es, Juan y Andrés). Por ello, se puede entender perfectamente que «visitó parajes muy bellos con él» expresa que «Juan visitó parajes muy bellos con Andrés». Ahora bien, el enunciado $\left(p_{4}\right)$ solo está coordinado respecto a $\left(p_{1}\right)$; por tanto, los pronombres del enunciado $\left(p_{4}\right)$ solo tendrán acceso a los referentes que aparezcan en $\left(p_{1}\right)$. Esto explica que el enunciado «Y, después, viajó a Turquía» se pueda interpretar solo como "Y, después, Juan viajó a Turquía», pero no es posible interpretar «Y, después, Andrés viajó a Turquía», aunque el referente de «Andrés» sí haya aparecido previamente.

Teniendo en cuenta estos principios generales, el referente del pronombre eso puede ser desentrañado en el discurso en una proporción de casos nada despreciable. La particularidad del pronombre eso es que, cuando este ocupa el contexto sintáctico propio de un SN (funciona como un SN), entonces su referente podrá ser de individuo $(x)$ o eventual (e):

11) Referente de individuo:

197 MJ: pues allí $\downarrow$ en el edificio de docentes/ en el aula de in[formática=] 198 Desc: $[(())]$

$199 \mathrm{MJ}:=$ una PLAGA de arañas/ y estuvimos una semana o dos sem-/ o dos seMAnas $\downarrow$ es que no me acuerdo/ sin dar clase de informática por eso.

(Corpus Valesco 2.0, Conversación 23, Intervenciones 195-203)

12) Referente de evento

$337 \mathrm{~A}:=$ distancias $\downarrow$ ni nad(a)/ pero vamos/ que me gusta mucho el agua// yy luego $\uparrow$ de pequeña $\uparrow$ sí que tenía una afición que era pues $\rightarrow$ pintar figuritas de estas de escayola $\uparrow /$ [quee me gustaba mucho=]

338 B:[sí $\downarrow /$ ya $\downarrow$ sí/ si]

$339 \mathrm{~A}:=$ y hacíaa $[(())]$

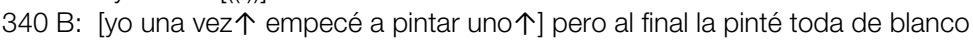

341 A: (RISAS)/ o sea que al final dijiste/ ya está bien

342 B: digo $\rightarrow$ ya no puedo más/y de blanco $\downarrow$

$343 \mathrm{~A}:\left[{ }^{\circ}\right.$ (qué risa) $\left.{ }^{\circ}\right]$

344 B: [no tenía] paciencia yo para eso.

(Corpus Valesco 2.0, Conversación 1, Intervenciones 337-344)

En el ejemplo de (11), el enunciado en el que aparece el pronombre eso mantiene una relación de coordinación (narración) con el enunciado previo ("pues allí, en el edificio de docentes, en el aula de informática, (hubo) una PLAGA de arañas») y, por tanto, el pronombre tiene acceso al referente expresado por «plaga de arañas». Por ello, el pronombre eso puede tomar ese referente de individuo. Por su parte, en el ejemplo de (12), el enunciado en el que aparece el pronombre eso mantiene una relación de subordinación (posiblemente, explicación) respecto al enunciado «sí que tenía una afición, que era, pues, pintar figuritas de estas de escayola». Por ello, pese a la distancia discursiva, el pronombre eso puede hacer referencia al evento "pintar figuritas de escayola» (es decir, "no tenía paciencia yo para pintar figuritas de escayola»), tal como predicen las distintas teorías semántico-pragmáticas.

Pero existen contextos en los que desentrañar el referente del pronombre eso no es fácilmente determinable ni por factores sintácticos ni factores semántico-pragmáticos. Este hecho es especialmente significativo en el caso de la estructura y eso.

13) $321 \mathrm{~A}:$ noo/ bueno $\rightarrow / /$ me gusta jugar/ no tengo mucha idea// reconozco que tengo más idea

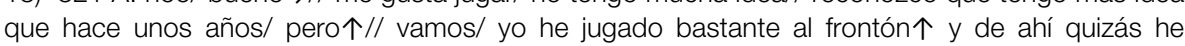


aprendido un poco pues al tenis [y eso=]

(Corpus Valesco 2.0, Conversación 1, Intervenciones 321)

En este contexto, el referente de eso queda abierto a diferentes interpretaciones. Puede referirse a diferentes deportes similares al tenis, puede referirse a las consecuencias no especificadas de jugar mejor, o puede referirse a otros elementos no fácilmente deducibles del contexto. Así pues, a priori, parecería que el discurso de los hablantes que utilizan expresiones como eso y, especialmente, y eso es ineficaz o irrelevante, en tanto que parece estar lleno de "inespecificidad", "ambigüedad"... Sin embargo, tal como se verá en el siguiente punto, incluso en estos casos, la proforma eso aporta un significado pragmático altamente relevante.

\section{EL COMPONENTE PRAGMÁTICO/DISCURSIVO: Y ESO}

Los hablantes tratan de conseguir que sus intervenciones sean lo más relevantes posibles; por tanto, ni siquiera en los casos en los que la búsqueda del referente de la proforma eso plantea serios problemas se ha de pensar que no tiene ningún valor. A nuestro juicio, aunque, en estos casos, el valor anafórico del elemento comienza a tener un valor secundario, la proforma eso no es un elemento sin significado, sino que su significado es, como mínimo, discursivo. El sentido de la proforma dependerá en buena medida de la posición del elemento y de la entonación.

En posición inicial de turno, el valor anafórico de la proforma todavía no queda desdibujado totalmente, tal como se puede apreciar:

14) $5 \mathrm{~A}$ : = y lo sacas/peroo si es cosa ya dee/ unn proyecto $\uparrow /$ que en ese tribunal le gusta o no le gusta $\uparrow$ pues entonces $\uparrow / /$ te lo aprueban o no te lo aprueban dependiendo dee§

$6 \mathrm{~B}$ : § o sea que [tenéis que presentar un proyecto $\uparrow=]$

7 A:[del gusto del tribunal (())]

$8 \mathrm{~B}:=$ y eso ¿en qué consiste $\downarrow$ ?

9 A: eel- ¿el proyecto?/ pues nada/ por ejemplo $\downarrow$ te dan unas bases sobree un aula $\downarrow$ oo sobre determinado colegio $\uparrow$ diciendo $\uparrow /$ suponiendo que tuviéramos tanto materiaal [para=]

(Corpus Valesco 2.0, Conversación 1, Intervenciones 5-8)

En este caso, parece sensato considerar que el referente de la proforma eso es el proyecto. El valor anafórico, por tanto, parece subsistir aquí. Pero junto a este valor, existe otro valor discursivo nada desdeñable de y eso: introduce un subtema del tema tratado, esto es, una pequeña digresión o aclaración, cuando va seguido de una pregunta. Este valor pragmático se puede rastrear en diversas ocasiones, aunque la pregunta se presente como mera pregunta confirmatoria (¿no?, ¿verdad?...):

15) $23 \mathrm{D}$ : ha entrao pasaoo $\uparrow$ pasaoo $/ / /$ él no ha podido frenaar $\uparrow$ aa-aa punto para-para meterse en la curvaa él se haa salido de curvaa y a vove- y ha vuelto a entrar cuando aquellos ya pasabaan $\uparrow / / /$ y es cuando el ha golpeao a los otros porque aquellos ya pasaban/ si tú los adelantaas/como vah a tropezar a alguien que has adelantaao/ eso es porque te has colaao y aquellos te han vuelto a pasar $\mathrm{y} / \mathrm{ttu}$ te has querido meterte otra vez/// ess un error de Lorenzo§ $24 \mathrm{P:} \S^{\circ}(\mathrm{si})^{\circ}$

25 D: la culpa $\uparrow$

26 P: es un error total///(2?)

27 D: y eso es protestaable ¿noo? ee- pero no- pero nunca los jueces nuncaa $\uparrow$

28 P: ${ }^{\circ}(\text { no })^{\circ}$ no porque no ha ocurrido nadaa $\uparrow$ noo $\uparrow$ no se ha caido ni nadaa/ peroo a lo mejor si se hubiese caidoo// quizá $\uparrow$ DoviZIOso/ porque estos son así/ eeh/ marrulleros hubiese metido bullaa/ al equipo///(2?5?) pocas vueltas

(Corpus Valesco 2.0, Conversación 15, Intervenciones 23-27)

Por tanto, se puede afirmar que la expresión y eso seguido de una pregunta a inicio de turno es un marcador pragmático de invitación a abrir un turno reactivo en el interlocutor y 
dicho valor parece mantenerse independientemente de si el referente del pronombre es evidente o no.

Este valor pragmático de la proforma puede aparecer cuando la expresión forma todo un acto; en este caso, el tonema ha de ser ascendente y afectar a toda la expresión:

16) 1 A: Ays $\uparrow$ / estoy más cansada

$2 \mathrm{~B}:$ ¿Y eso?

3 A: bua imagínate/ to(do) el día en clase.

(Corpus Valesco 2.0, Conversación 33, Intervenciones 1-3)

Por el contrario, cuando aparece a principio de turno con un tonema ascendente marcado, el valor pragmático parece ser el contrario: es un indicador de toma o mantenimiento del turno, tal como se muestra en el siguiente ejemplo:

17) 90 B: [dice es que metí un golazo deel medio del campo y metí otro golazo de cabezaa y me dijeron (( )) ((ino sabíamos)) que eras tan bueno! / y yo pensé mira hijo lo único que has hecho en to(d)a tu vida $/ / /(1,01)(($ desde el)) fútbol me refiero

91 A: ((yaa))

92 B: y eso $\downarrow$ sí sí y entonces claro se picaba muchísimo porque le decíamos Pepe tío ///(1,31) nos tenemos que salir todos del campo y éliva no que yoo aún no estoy cansao! ((y dije)) mira que entre que eres malo y que nos tenemos que salir to(do)s del campo isalte tío! §

$93 \mathrm{~A}: \S[(\mathrm{RISAS})]$ lo de que eres malo te lo podías ahorraar [(RISAS)]

(Corpus Valesco 2.0, Conversación 44, Intervenciones 90-93)

Si la expresión y eso se encuentra en medio del turno, iniciando un acto y seguido de una pregunta, su valor pragmático no dista del anteriormente señalado. Quizás sí se haya de indicar que su valor fórico no parece estar tan claro ya en este contexto:

18) 140 B: porque cuando seas $\uparrow / /$ profesora oficial $\rightarrow$

141 A: PROFESORA OFICIAL $\downarrow(())$ tendré que hacer yo ahí una estructuración de lo que tengo [que hacer]

142 B:[no] pero eso ya / y eso ¿qué haces luego? ¿vas cogiendoo/ el currículum $\uparrow$ o es- eso es tipo de sustituciones $\uparrow$ oo?

(Corpus Valesco 2.0, Conversación 1, Intervenciones 90-93)

Si la entonación y el contexto no son los señalados, la proforma eso parece guardar todavía su valor anafórico y su valor pragmático no está tan claro, como ocurre en:

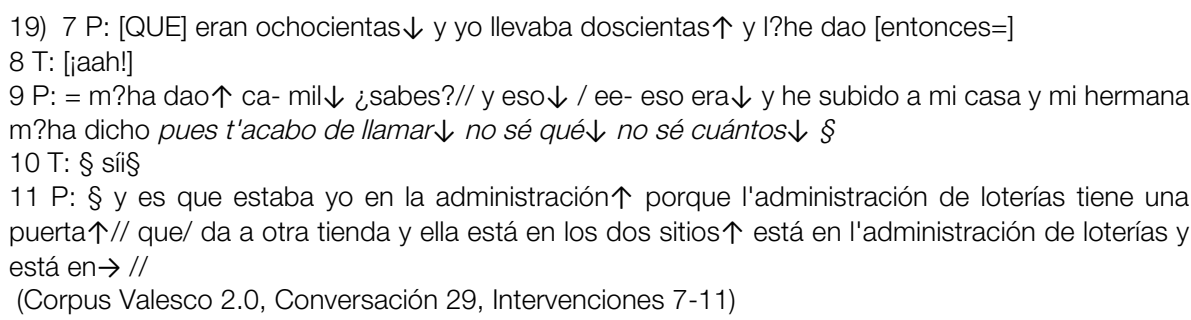

Finalmente, la expresión y eso puede aparecer a final del turno con cualquier entonación. En este caso, esta expresión parece indicar el cese del turno del hablante.

20)

a. Entonación ascendente

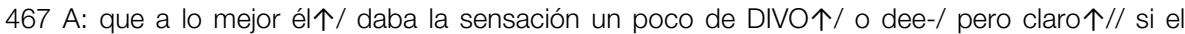
intentaba cada día mejorarse $\uparrow$ [y eso $\uparrow$ ]

468 B: [es que] es eso $\downarrow /$ es un afán de superación [que él mismo tenía]

(Corpus Valesco 2.0, Conversación 1, Intervenciones 467-468)

b. Entonación de suspensión

154 B: tío $\downarrow$ mira jvete/ a la PUTA MIERDA!// ¿vale?

155 C: ${ }^{\circ}$ (no te enfades P.) ${ }^{\circ}{ }^{\circ}$ (no te enfades) ${ }^{\circ}$ 
156 B: pues no/ no [está claro]

157 A: [iqué va!] yo me- yo me quiero comprar $\downarrow$ una/ para cuando entre en clase y eso $\rightarrow$

158 C: sí

159 B: ya tía/ oye ¿se pueden grabar $\downarrow$ a los profesores $\downarrow /$ la clase?

(Corpus Valesco 2.0, Conversación 24, Intervenciones 154-159).

c) Tonema descendente

22 F: iy es que llego hecha una mierda!/ llego a las diez y media $\uparrow$ y llego hecha una mierda // pero el profesor estee me deja hecha una mierda $\downarrow$ encima si por la tarde no hago la siesta $\uparrow / /$ es que tía noo/ yo no aguanto $\downarrow$ llega la última hora y ya me estoy cayendo allii /en medio $\downarrow$ ¿sabes?/ y eso $23 \mathrm{H}:$ [sí/como yo]/ que me duermo en clase [(RISAS)]

(Corpus Valesco 2.0, Conversación 26, Intervenciones 22-24).

Como se puede observar en estos tres casos, aunque el valor discursivo es bastante claro (cese del turno), en cambio, el referente del pronombre ya no es tan evidente. De hecho, habría serias dificultades para desentrañar el referente contextualmente. Por tanto, se podría decir que la posición que ocupa la expresión y eso en el discurso está relacionado con la opacidad referencial de la proforma, pero no así con el nítido valor pragmático que adopta (en cuyo caso la entonación parece ser ya un elemento secundario). Además, hay que observar que, en este contexto, el valor fraseológico de y eso parece mucho más consumado que en los ejemplos anteriores en los que y eso no ocupa una posición final ${ }^{3}$. Por tanto, podríamos ya estar hablando de un elemento que se ha especializado en delimitar el final de turno.

\section{CONCLUSIONES}

Tal como se ha pretendido mostrar en el presente trabajo, la proforma eso es un elemento que no es inespecífico o ambiguo, sino que tiene una función muy clara en el discurso. Dicho elemento puede hallar su referente en el discurso previo bien mediante la información gramatical (contexto sintáctico) o bien mediante la información semántico-discursiva (mediante las relaciones retóricas). Pero incluso, en aquellos casos en los que a dicho elemento no se le puede asignar un referente claro, como ocurre con suma frecuencia en la expresión y eso, la proforma presenta una función discursiva que no puede ser ignorada, pues es este valor el que lo hace obligatorio discursivamente. Así por ejemplo, la expresión y eso a final de turno presenta un significado menos ligado a la foricidad y más marcadamente procedimental. Por ello, la proforma eso nunca es superficial, innecesaria o irrelevante, a nuestro juicio.

A nuestro entender, sería conveniente ampliar el estudio con otras expresiones que incluyeran la proforma analizada (v.gr.: y eso que, por eso, eso sí, pues eso, eso es...) y delimitar sus distintos sentidos en el discurso (¿son parejos? ¿aparecen dichos significados en los mismos contextos discursivos?...).

\section{BIBLIOGRAFÍA}

Asher, Nicholas (1998): «Discourse Structure and the Logic of Conversation». First Workshop on Non-narrative Text Structure. Austin. Elsevier: 1-28. [en línea] http://www.utexas.edu/cola/depts/philosophy /faculty/asher/.../DSLC.ps [consulta: 22-52016].

\footnotetext{
${ }^{3}$ Si se toma un ejemplo como (16), el elemento eso se puede sustituir todavía por un elemento nominal, ¿Y ese estado/cansancio? o toda una oración, ¿Y por qué estás así? Ahora bien, en los ejemplos en los que ocupa la posición final, la recuperación del referente es más difícil; a nuestro juicio, se debería por el hecho de que en esta posición, el valor pragmático-discursivo se ha consumado. 
Asher, Nicholas y Alex Lascarides (2003): Logics of conversation, Cambridge, Cambridge University Press.

Grupo Val.Es.Co (2014): «Las unidades del discurso oral», Estudios de Lingüística del Español 35 (2014), 13-73.

Hengeveld, Kees y J. Lachlam Mackenzie (2008): Functional Discourse Grammar. Oxford. Oxford University Press.

Hengeveld, Kees y J. Lachlam Mackenzie (2010): «Functional Discourse Grammar». The Oxford Handbook of Linguistic Analysis. Bernd Heine y Heiko Narrog, ed., Oxford, Oxford University Press, 367-400.

Mann, William C. y Sandra A. Thompson (1987): «Rhetorical Structure Theory: a framework for the analysis of texts", Papers in Pragmatics, 1, 79-105.

Mann, William C. y Sandra A. Thompson (1988): «Rhetorical Structure Theory: toward a functional theory of text organization», Text (8), 3, 243-281.

Prasad, Rashmi, Nikhil Dinesh, Alan Lee, Aravind Joshi, y Bonnie Webber (2007): «Attribution and its annotation in the Penn Discourse TreeBank», Traitement Automatique des Langues, Special Issue on Computational Approaches to Document and Discourse, 47(2), [en línea] https://www.seas.upenn.edu/ pdtb/papers/pr asad-etal-attribution-acl06.pdf [consulta: 2-62016]. 1-8.

Prasad, Rashmi, Aravind Joshi y Bonnie Webber (2010): "Realization of Discourse Relations by Other Means: Alternative Lexicalizations", Proceedings of the 23rd International Conference on Computational Linguistics (COLING 2010), Pekín, [en línea] http://www.seas.upenn.edu/ rjprasad/papers /prasad-etal-altlex-coling-2010.pdf [consulta: 2-6-2016] 1-9.

Rashmi Prasad, Nikhil Dinesh, Alan Lee, Eleni Miltsakaki, Livio Robaldo, Aravind Joshi y Bonnie Webber (2008): «The Penn Discourse TreeBank 2.0", Proceedings of the 6th International Conference on Language Resources and Evaluation (LREC 2008), [en línea]

https://www.seas.upenn.edu/ pdtb/papers/p dtb-Irec08.pdf [consulta: 2-6-2016], 1-8.

Sperber, Dan y Deirdre Wilson (1986 [1994]), La Relevancia, Madrid, Visor.

Webber, Bonnie, Mathew Stone, Aravind Joshi, y Alistair Knott (1994): «Anaphora and Discourse Structure». Association for Computational Linguistics, [en línea] https://www.cs.rutgers.edu/ mdstone/pubs/a ds2.pdf [consulta: 2-6-2016], 1-45. 